\title{
Pulsars: implications from Fermi LAT observations and future prospects
}

\section{Lucas Guillemot*, on behalf of the Fermi LAT Collaboration, and the Pulsar Search and Timing Consortia}

Laboratoire de Physique et Chimie de l'Environnement et de l'Espace - Université d'Orléans/CNRS, F-45071 Orléans, France

Station de radioastronomie de Nançay, Observatoire de CNRS/INSU, F-18330 Nançay, France,

E-mail: lucas.guillemotecnrs-orleans.fr

\begin{abstract}
Gamma-ray observations with the Fermi Large Area Telescope (LAT) have revealed significant pulsations from more than 200 young and recycled, millisecond pulsars. These observations have demonstrated that pulsars are by far the largest source class in the Galactic plane at GeV energies, and more gamma-ray pulsars are still being revealed by the LAT. In these proceedings I give an overview of the main results from Fermi LAT pulsar observations. I also discuss some of the implications of these results in terms of population statistics and high-energy pulsar emission properties.
\end{abstract}

7th Fermi Symposium 2017

15-20 October 2017

Garmisch-Partenkirchen, Germany

\footnotetext{
* Speaker.
} 


\section{Gamma-ray pulsars in 2017}

The Large Area Telescope (LAT), main instrument on the Fermi observatory, is sensitive to photons with energies between $20 \mathrm{MeV}$ and more than $300 \mathrm{GeV}$ (see [1] for a detailed description). Its unprecedented sensitivity, excellent timing accuracy (LAT timestamps are good to better than 1 $\mu$ s relative to UTC, see [2]), and its survey observation strategy make the Fermi LAT a wonderful instrument for observing and studying gamma-ray pulsars in the GeV domain.

Since the launch of Fermi in 2008, observations with the LAT have revolutionized our view of the population of gamma-ray pulsars. From fewer than ten firm pulsar detections and a few marginal ones before the mission (thanks to observations made by AGILE, EGRET, and previous instruments; e.g. [3, 4]), we now stand at a total of 210 pulsars $^{1}$ firmly detected in gamma rays with the LAT, a non-negligible fraction of the $\sim 2600$ pulsars known currently at any wavelengths [5]. Figure 1 shows a map of the LAT-detected pulsars in Galactic coordinates. As can be seen from this map, a high fraction of low Galactic latitude LAT sources are "normal" pulsars (i.e., pulsars with spin periods $P$ ranging from $\simeq 50 \mathrm{~ms}$ to $10 \mathrm{~s}$, and large spin-down rates $\dot{P}$ ), similar to those known prior to Fermi. Interestingly, the LAT also detects many pulsars at high Galactic latitudes. Most of these pulsars are "millisecond" pulsars (MSPs, with spin periods $P$ below $\simeq 30 \mathrm{~ms}$ and very low $\dot{P}$ values), thought to have spun-up to millisecond periods by the accretion of matter and transfer of angular momentum from a binary companion [6,7]. The vast majority of gamma-ray MSPs are in the Galactic disk, and a few are in globular clusters [8,9].

Figure 2 shows the locations of the LAT-detected pulsars in the classical $P-\dot{P}$ diagram. Also displayed in the diagram are lines of constant spin-down power $\dot{E}=4 \pi^{2} I \dot{P} / P^{3}$, assuming a value of $10^{45} \mathrm{~g} \mathrm{~cm}^{2}$ for the neutron star moment of inertia, $I$. We see from the diagram that the pulsars seen by the LAT are energetic, and have $\dot{E}$ values above $\gtrsim 10^{33} \mathrm{erg} \mathrm{s}^{-1}$. Pulsar distances, which are not represented in the diagram, also play a key role in gamma-ray detectability. With a few exceptions (e.g., pulsars in globular clusters, PSR B0540-69 in the Large Magellanic Cloud, etc.), LAT pulsars are also nearby objects. From the black and grey dots in Figure 2 we see that many pulsars with $\dot{E}$ values lower than $10^{33} \mathrm{erg} \mathrm{s}^{-1}$ have been searched for gamma-ray pulsations in the LAT data, to no avail. The spin-down power threshold of $\sim 10^{33} \mathrm{erg} \mathrm{s}^{-1}$ is therefore an empirical "deathline" for gamma-ray emission.

For gamma-ray pulsars with measured distances it is possible to calculate the gamma-ray luminosity $L_{\gamma}$, and compare it with the spin-down power $\dot{E}$. The latter quantity can be seen as the total energy budget that pulsars can convert into, for instance, electromagnetic radiation. Figure 3 displays the luminosities of LAT-detected pulsars as a function of $\dot{E}$. Observed luminosities span almost five orders of magnitude, and spin-down powers span about six. The two quantities are clearly correlated, although luminosity values in a given $\dot{E}$ range are often highly dispersed. One of the key questions to address is that of the exact relationship between $L_{\gamma}$ and $\dot{E}$. Getting more accurate distance and proper motion estimates (transverse proper motions can make apparent and intrinsic $\dot{P}$ values differ significantly, see [12]) e.g. from timing measurements, beaming constraints from theoretical studies, and understanding outliers (such as PSR J1024-0719 whose apparent

\footnotetext{
${ }^{1}$ See https://confluence.slac.stanford.edu/display/GLAMCOG/Public+List+of+ LAT-Detected+Gamma-Ray+Pulsars for a publicly-available list of LAT-detected pulsars.
} 


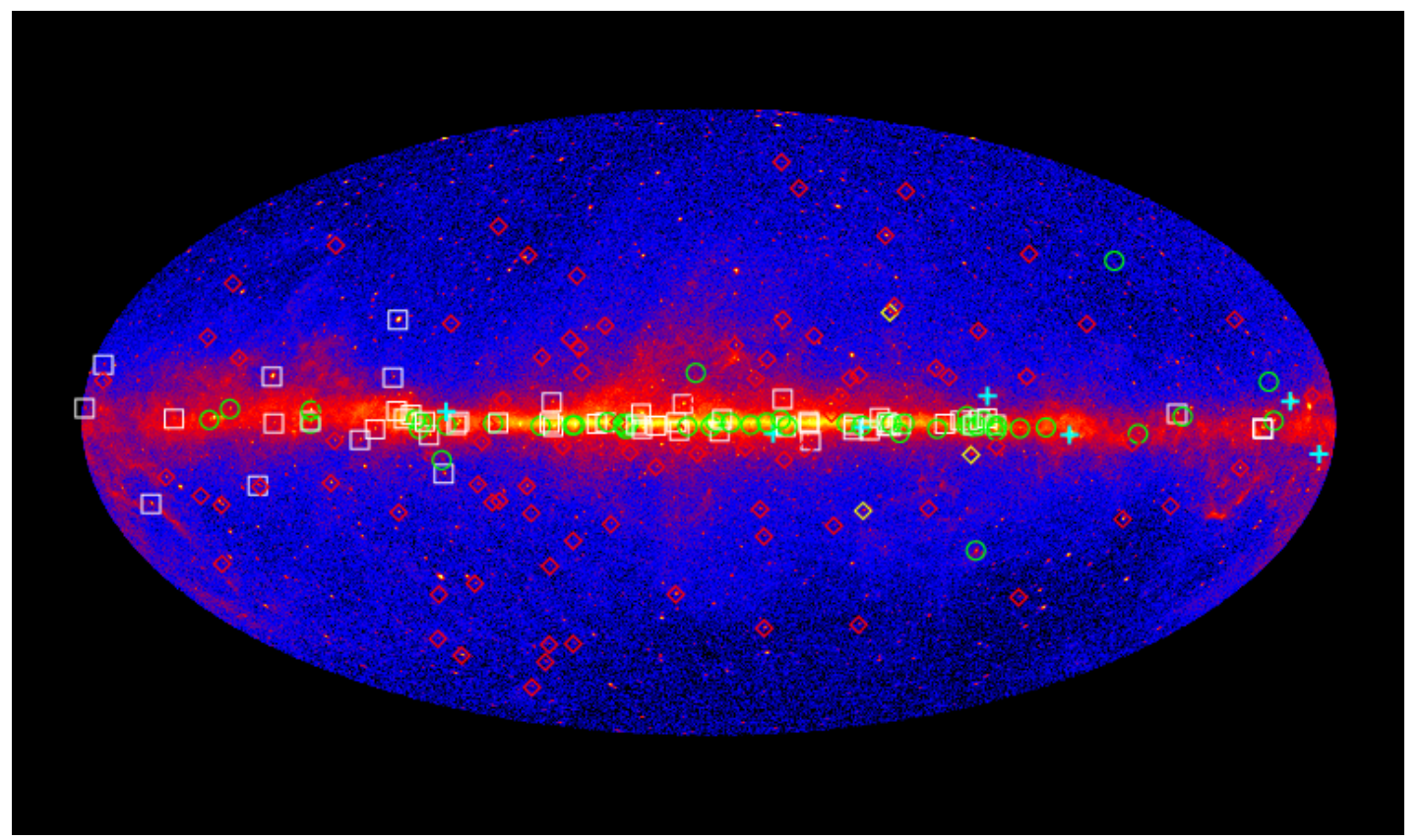

Figure 1: Map showing Pass 8 Fermi LAT all-sky survey data in Galactic coordinates, with the locations of LAT-detected pulsars. Green circles indicate normal, radio- and/or X-ray-loud pulsars. Cyan crosses show the pulsars already seen by EGRET, and white squares correspond to gamma-ray-selected normal pulsars, i.e., pulsars discovered in blind searches of the LAT data (see Section 2). Red diamonds are millisecond pulsars that were first known from radio observations, while yellow diamonds correspond to MSPs found by blind searching the LAT data, similarly to gamma-ray-selected normal pulsars.

spin-down rate is severely affected by acceleration around a nearby main sequence star, see [13]) will be crucial for addressing this problem.

\section{Gamma-ray pulsar detections and discoveries}

A plot of the number of LAT-detected pulsars as a function of time from early 2009 (i.e., soon after Fermi's launch) to mid-2016, split into different pulsar sub-populations, is shown in Figure 4. Interestingly, the total number of LAT-detected pulsars has so far increased almost linearly. Sudden increases in the detection rate can have multiple causes: LAT data improvements (e.g. Pass 8), bursts of pulsar discoveries from gamma-ray blind searches, etc. Up to now (the numbers quoted in the rest of this section may slightly differ from those in Figure 4), a total of 58 normal pulsars known to emit radio and/or X-ray emission have been detected by the LAT. A recent and particularly interesting example is that of PSR B0540-69 in the Large Magellanic Cloud [14]. Gamma-ray pulsations from this pulsar are relatively faint, but its very large distance for a gamma-ray pulsar of $\sim 50 \mathrm{kpc}$ makes B0540-69 the most luminous gamma-ray pulsar known at present. Smith et al. (2017) [11] also recently reported the detection of six recently-discovered pulsars with the LAT, some of them exhibiting very faint gamma-ray pulsations. A "Pulsar Timing Consortium" was organized before the launch of Fermi, to provide the LAT collaboration with contemporaneous timing solutions for as many pulsars as possible [15]. Most of these pulsars have been detected 


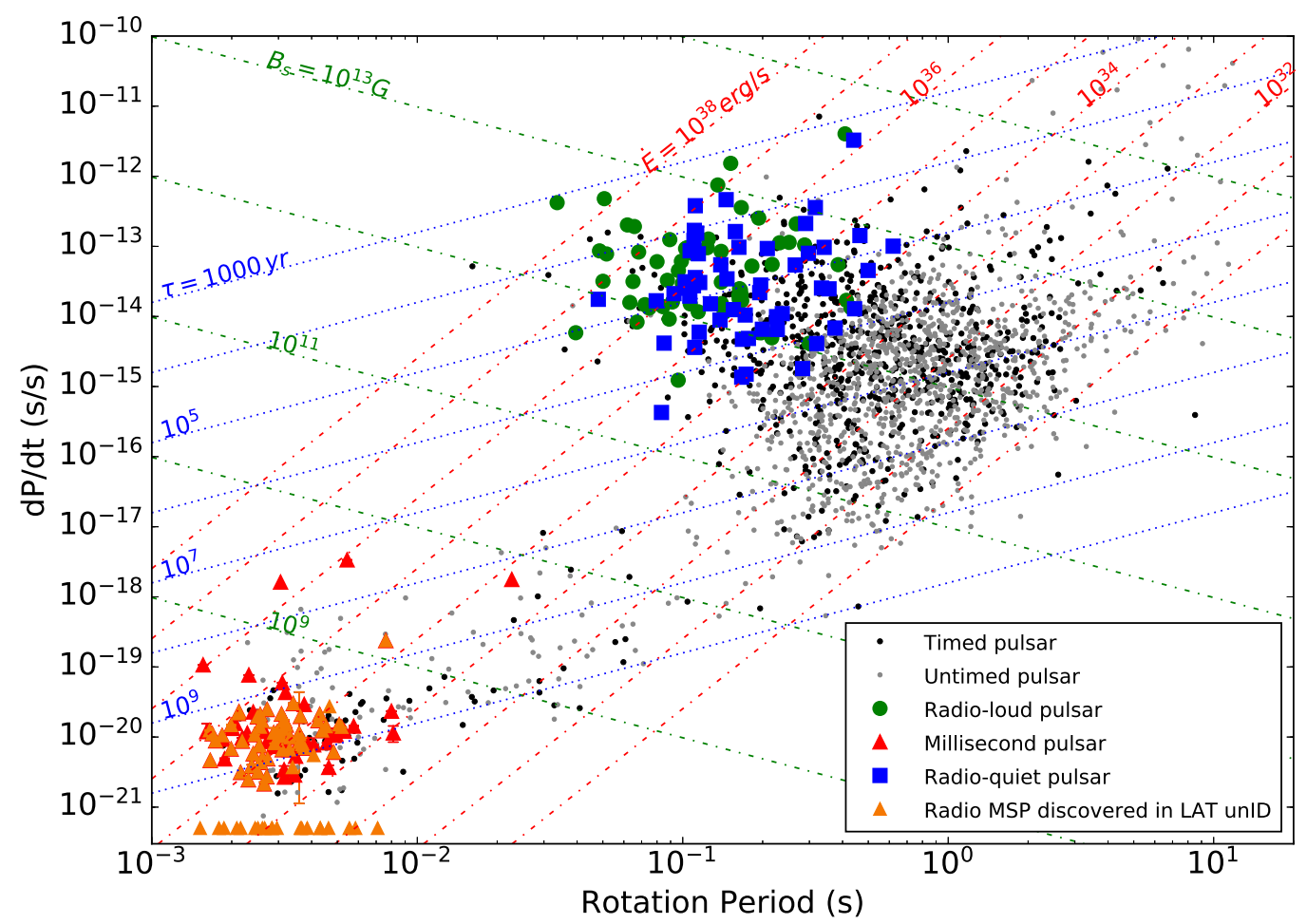

Figure 2: Period $P$ - spin-down rate $\dot{P}$ diagram showing the currently known population of pulsars, and representing LAT-detected pulsars as colored symbols. Black dots correspond to pulsars for which gammaray pulsations have been searched for in the LAT data. Grey dots are pulsars that have not been searched for gamma-ray pulsations. Orange triangles display radio MSPs found at the locations of LAT sources and with no gamma-ray pulsations detected to date, although most of them are expected to be detectable as pulsed sources of gamma rays once accurate timing solutions covering several years of LAT data will become available. The line of orange triangles at the bottom left of the diagram are radio MSPs discovered in LAT sources, with currently unknown or unpublished spin-down rates. Lines of constant characteristic age $\tau$, surface magnetic field $B_{s}$, and spin-down power $\dot{E}$ are also displayed. This figure is updated from $2 \mathrm{PC}$, the second Fermi LAT catalog of gamma-ray pulsars [10], and is adapted from [11].

using timing solutions provided by this consortium, whose contribution is also vital for probing the gamma-ray emission "deathline" (as can be seen from the black and grey dots in Figure 2).

An increasing population of normal "gamma-ray-selected" pulsars, comprising 57 objects at present, were not known prior to Fermi and were discovered by blind searching the LAT data for pulsars (e.g. [16, 17]). Due to the paucity of LAT photons, blind searches for gamma-ray pulsars with no (or limited) information about the precise sky location and rotational parameters are extremely computer intensive. Efficient techniques have been developed to tackle this problem [18, 19], but still require large amounts of computing power. Some of the newly-discovered gamma-ray pulsars are very interesting: PSR J1838-0537 has suffered one of the largest glitches ever observed, which has made its discovery difficult [20]. PSR J1208-6238 [21], discovered in 


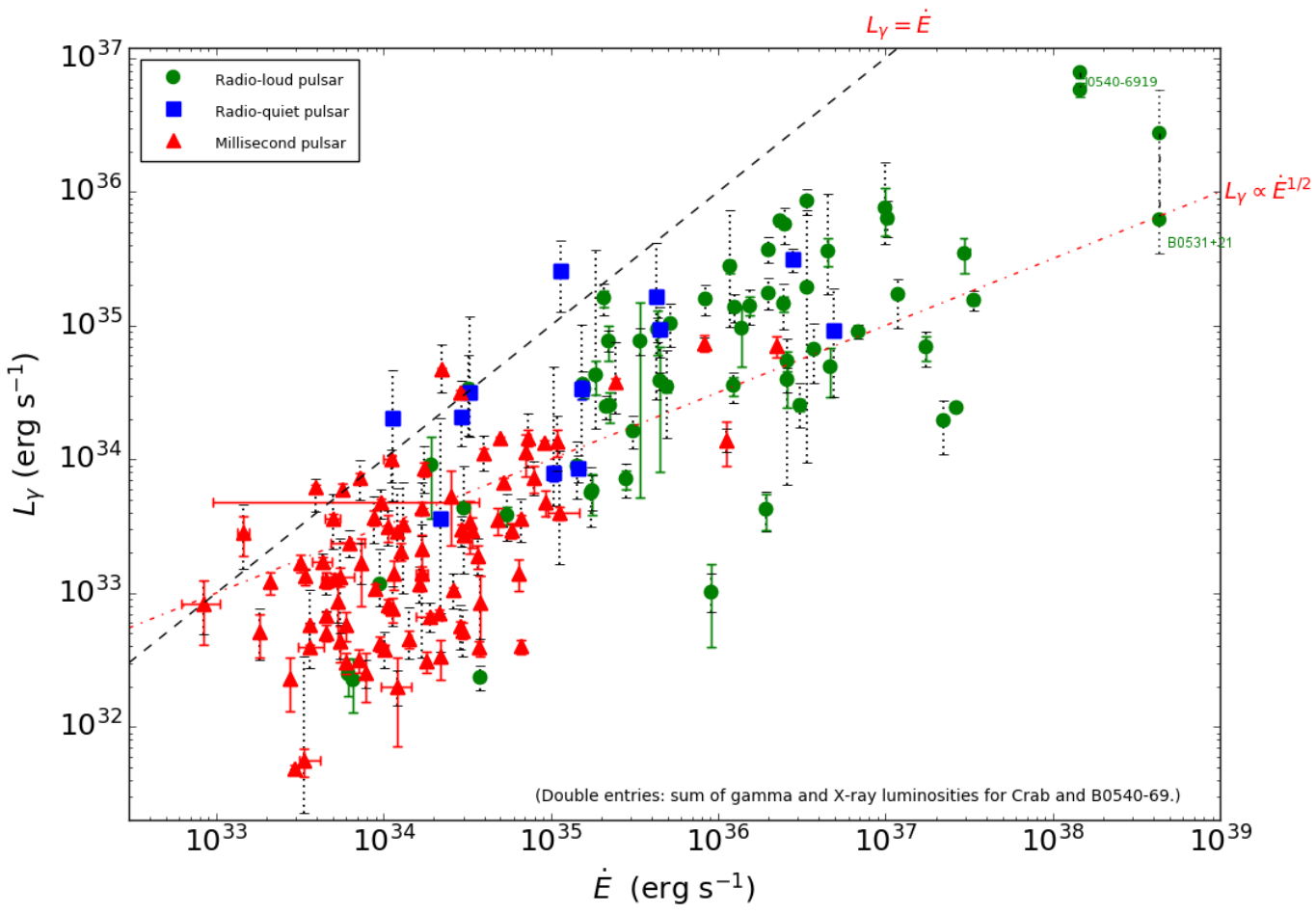

Figure 3: Gamma-ray luminosity as a function of spin-down power, for the LAT-detected pulsars. The colors and markers are the same as in Figure 2. Luminosities were calculated as $L_{\gamma}=4 \pi f_{\Omega} h d^{2}$, where $d$ is the distance, $h$ is the gamma-ray energy flux, and $f_{\Omega}$ is a geometrical correction factor. For this plot we used $f_{\Omega}=1$ for all pulsars. This figure is updated from 2PC. Figure courtesy of David A. Smith.

a blind survey of the LAT data with the Einstein@Home volunteer computing system, has a measurable braking index giving us valuable insights into the pulsar's braking mechanism. Braking indices have only been measured for about ten pulsars so far. Clark et al. (2017) [22] and Wu et al. (2017, submitted) present the properties of 13 pulsars found in a recent survey with Einstein@ Home. Similarly to the rest of the population of "gamma-ray-selected" normal pulsars, very few of them are also detected in radio, in spite of deep radio observations. Without the LAT, it is thus likely that these radio-quiet or radio-faint pulsars would have remained unknown for many years. Blind searches for pulsars in the LAT data will continue in the future, and will for example use unassociated sources from the upcoming 4FGL catalog as their search targets.

Finally, one of the most significant results of the Fermi LAT was mostly unexpected before the launch of Fermi. Although no gamma-ray MSP had been firmly detected to emit gamma-ray pulses at the beginning of the mission, MSPs now represent almost half of the total LAT pulsar population (see Figure 4). The majority of LAT-detected MSPs are in the Galactic disk (e.g. [23]). Some MSPs in globular clusters are detected individually, as pulsed sources of gamma rays [8,9]. Most of the MSPs seen by the LAT were first known from radio observations, and were either found in "traditional" radio pulsar surveys or in searches for radio pulsars at the locations of LAT sources with no known associations, by the telescopes involved in the Pulsar Search Consortium (PSC). The 


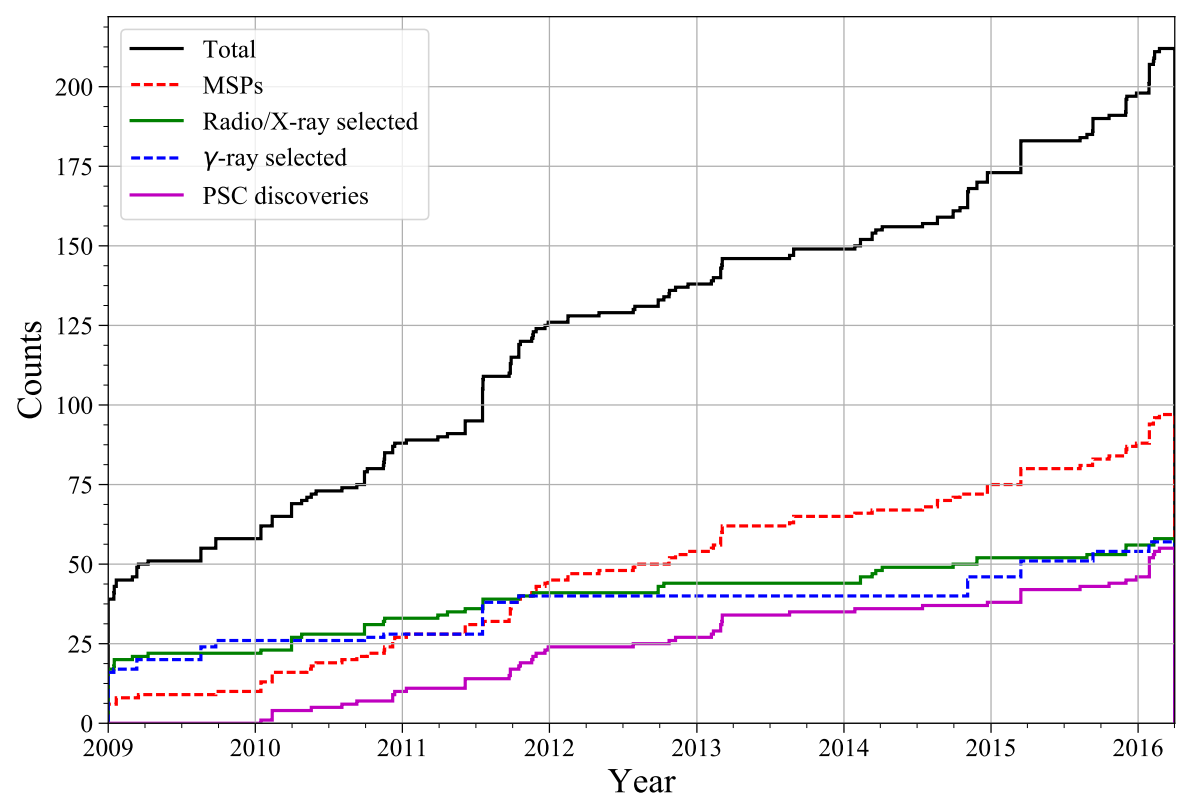

Figure 4: Number of LAT-detected pulsars as a function of time, until April 2016. The green line corresponds to radio- or X-ray-selected normal pulsars, while the number of gamma-ray-selected normal pulsars is represented by the dashed blue line. The dashed red line corresponds to MSPs. The total number of LAT-detected pulsars, corresponding to the sum of these three sub-populations, is shown by the black line. Finally, the magenta line shows the number of pulsars found in LAT sources by the Pulsar Search Consortium. Most of these pulsars are MSPs, that are eventually detected as pulsed sources of gamma rays, once a good timing solution becomes available.

latter searches have been amazingly successful, with more than 80 pulsars discovered so far, most of them being MSPs. A large fraction of these new MSPs are black widows or redback systems. Some MSPs are very stable rotators and are being added to Pulsar Timing Arrays (e.g., [24]), which aim at detecting and characterizing very low-frequency (typically, $\sim 1 \mathrm{nHz}$ ) gravitational waves, by analyzing the radio pulse arrival times from a population of stable MSPs distributed across the sky. Without any prior information on the orbital parameters, MSPs, which often reside in binary systems, are extremely difficult to find in direct blind searches of the LAT data. The black widow pulsar PSR J1311-3430, whose orbital parameters had been constrained with sufficient accuracy by optical observations, was the first MSP ever found in blind gamma-ray searches [25]. More recently, two isolated MSPs have been discovered, with Einstein@Home (Clark et al. 2017, submitted). A more detailed description of searches for millisecond pulsars in LAT sources and of the numerous discoveries can be found in the contribution by C. Clark, these proceedings. Similarly to normal pulsars, blind searches will likely uncover more MSPs; and PSC telescopes will also likely continue to discover more radio MSPs in LAT sources with no known counterparts. 


\section{Pulsar emission properties and the third catalog of LAT pulsars}

Figure 3 shows that, for many LAT-detected pulsars, gamma-ray emission often represents a large fraction of the total energy output from pulsars. This is particular true for low- $\dot{E}$ pulsars, which often have efficiency values $\eta=L_{\gamma} / \dot{E}$ in excess of $10 \%$ of more. High-energy gamma rays therefore enable us to probe the primary particle acceleration processes that take place around neutron stars. Gamma-ray emission spectra are usually well fitted by exponentially cut off power laws, as is expected for emission dominated by curvature radiation in the radiation-reaction limited regime (see [10] for the distribution of pulsar spectral properties in gamma rays). Some bright pulsars exhibit sub-exponential cutoffs, caused by the blending of curvature radiation with varying spectra across the rotation. Pulse profiles in $\mathrm{GeV}$ gamma rays often comprise two narrow peaks separated in phase, with the first emission peak lagging the main radio peak, if radio emission is also seen. Such radio/gamma-ray pulse profiles suggest that the corresponding emission regions are separated, and that the gamma rays are produced at high altitudes in the magnetosphere or beyond the light cylinder (the radial distance from the neutron star surface at which the rotational velocity reaches the speed of light). Exceptions to this rule exist, in particular for MSPs, which sometimes display closely aligned radio and gamma-ray emission profiles [26]. Recent, comprehensive reviews of LAT pulsar results and modeling efforts can be found in [27, 28].

In 2013, the Fermi LAT Collaboration published "2PC", the second Fermi LAT catalog of gamma-ray pulsars [10], which tabulated the temporal and spectral emission properties of 117 pulsars detected by the LAT during the first three years of the mission. 2PC itself followed 1PC, published in 2010 [29], which presented the properties of 46 gamma-ray pulsars seen using the first six months of LAT data. The LAT Collaboration has started preparing 3PC, which will summarize the characteristics of the $>200$ pulsars so far seen by the LAT. 3PC will include careful determinations of the pulsars' gamma-ray temporal and spectral properties, fits of their pulse profiles, gamma-ray luminosities, etc., as was done for 2PC. Pulsar timing solutions and other ancillary files needed for reproducing the results will be distributed publicly, for usage by the community.

\section{Acknowledgements}

I am grateful to the organizers of this wonderful conference, for giving me to opportunity to give this presentation on Fermi LAT observations of pulsars.

The Fermi-LAT Collaboration acknowledges support for LAT development, operation and data analysis from NASA and DOE (United States), CEA/Irfu and IN2P3/CNRS (France), ASI and INFN (Italy), MEXT, KEK, and JAXA (Japan), and the K.A. Wallenberg Foundation, the Swedish Research Council and the National Space Board (Sweden). Science analysis support in the operations phase from INAF (Italy) and CNES (France) is also gratefully acknowledged.

\section{References}

[1] W. B. Atwood, A. A. Abdo, M. Ackermann, W. Althouse, B. Anderson, M. Axelsson et al., The Large Area Telescope on the Fermi Gamma-Ray Space Telescope Mission, ApJ 697 (June, 2009) 1071-1102, [0 902 . 1089 ]. 
[2] A. A. Abdo, M. Ackermann, M. Ajello, J. Ampe, B. Anderson, W. B. Atwood et al., The on-orbit calibration of the Fermi Large Area Telescope, Astroparticle Physics 32 (Oct., 2009) 193-219, [0904.2226].

[3] D. J. Thompson, Gamma ray astrophysics: the EGRET results, Reports on Progress in Physics 71 (Nov., 2008) 116901, [0811.0738].

[4] J. P. Halpern, F. Camilo, A. Giuliani, E. V. Gotthelf, M. A. McLaughlin, R. Mukherjee et al., Discovery of High-Energy Gamma-Ray Pulsations from PSR J2021+3651 with AGILE, ApJ Lett. 688 (Nov., 2008) L33, [0810.0008].

[5] R. N. Manchester, G. B. Hobbs, A. Teoh and M. Hobbs, The Australia Telescope National Facility Pulsar Catalogue, Astron. J. 129 (Apr., 2005) 1993-2006, [astro-ph/ 0412641 ].

[6] G. S. Bisnovatyi-Kogan and B. V. Komberg, Pulsars and close binary systems, Sov. Ast. 18 (Oct., 1974) 217.

[7] M. A. Alpar, A. F. Cheng, M. A. Ruderman and J. Shaham, A new class of radio pulsars, Nature 300 (Dec., 1982) 728-730.

[8] P. C. C. Freire, A. A. Abdo, M. Ajello, A. Allafort, J. Ballet, G. Barbiellini et al., Fermi Detection of a Luminous $\gamma$-Ray Pulsar in a Globular Cluster, Science 334 (Nov., 2011) 1107, [1111. 3754 ].

[9] T. J. Johnson, L. Guillemot, M. Kerr, I. Cognard, P. S. Ray, M. T. Wolff et al., Broadband Pulsations from PSR B1821-24: Implications for Emission Models and the Pulsar Population of M28, ApJ 778 (Dec., 2013) 106, [1310.1852].

[10] A. A. Abdo, M. Ajello, A. Allafort, L. Baldini, J. Ballet, G. Barbiellini et al., The Second Fermi Large Area Telescope Catalog of Gamma-Ray Pulsars, ApJ Suppl. 208 (Oct., 2013) 17, [1305.4385].

[11] D. A. Smith, L. Guillemot, M. Kerr, C. Ng and E. Barr, Gamma-ray pulsars with Fermi, ArXiv e-prints (June, 2017), [1706.03592].

[12] L. Guillemot, D. A. Smith, H. Laffon, G. H. Janssen, I. Cognard, G. Theureau et al., The gamma-ray millisecond pulsar deathline, revisited. New velocity and distance measurements, A\&A 587 (Mar., 2016) A109, [1601.05987].

[13] C. G. Bassa, G. H. Janssen, B. W. Stappers, T. M. Tauris, T. Wevers, P. G. Jonker et al., A millisecond pulsar in an extremely wide binary system, MNRAS 460 (Aug., 2016) 2207-2222, [1604 . 00129 ].

[14] Fermi LAT Collaboration, M. Ackermann, A. Albert, L. Baldini, J. Ballet, G. Barbiellini et al., An extremely bright gamma-ray pulsar in the Large Magellanic Cloud, Science 350 (Nov., 2015) 801-805.

[15] D. A. Smith, L. Guillemot, F. Camilo, I. Cognard, D. Dumora, C. Espinoza et al., Pulsar timing for the Fermi gamma-ray space telescope, A\&A 492 (Dec., 2008) 923-931, [0810.1637].

[16] A. A. Abdo, M. Ackermann, M. Ajello, B. Anderson, W. B. Atwood, M. Axelsson et al., Detection of 16 Gamma-Ray Pulsars Through Blind Frequency Searches Using the Fermi LAT, Science 325 (Aug., 2009) 840, [1009.0748].

[17] H. J. Pletsch, L. Guillemot, B. Allen, M. Kramer, C. Aulbert, H. Fehrmann et al., Discovery of Nine Gamma-Ray Pulsars in Fermi Large Area Telescope Data Using a New Blind Search Method, ApJ 744 (Jan., 2012) 105, [1111.0523].

[18] W. B. Atwood, M. Ziegler, R. P. Johnson and B. M. Baughman, A Time-differencing Technique for Detecting Radio-quiet Gamma-Ray Pulsars, ApJ Lett. 652 (Nov., 2006) L49-L52. 
[19] H. J. Pletsch and C. J. Clark, Optimized Blind Gamma-Ray Pulsar Searches at Fixed Computing Budget, ApJ 795 (Nov., 2014) 75, [1408.6962].

[20] H. J. Pletsch, L. Guillemot, B. Allen, M. Kramer, C. Aulbert, H. Fehrmann et al., PSR J1838-0537: Discovery of a Young, Energetic Gamma-Ray Pulsar, ApJ Lett. 755 (Aug., 2012) L20, [1207. 5333 ].

[21] C. J. Clark, H. J. Pletsch, J. Wu, L. Guillemot, F. Camilo, T. J. Johnson et al., The Braking Index of a Radio-quiet Gamma-Ray Pulsar, ApJ Lett. 832 (Nov., 2016) L15, [1611. 01292].

[22] C. J. Clark, J. Wu, H. J. Pletsch, L. Guillemot, B. Allen, C. Aulbert et al., The Einstein@Home Gamma-ray Pulsar Survey. I. Search Methods, Sensitivity, and Discovery of New Young Gamma-Ray Pulsars, ApJ 834 (Jan., 2017) 106, [1611.01015].

[23] L. Guillemot, M. Kramer, T. J. Johnson, H. A. Craig, R. W. Romani, C. Venter et al., Fermi LAT Pulsed Detection of PSR J0737-3039A in the Double Pulsar System, ApJ 768 (May, 2013) 169, [1303.7352].

[24] M. Kramer and D. J. Champion, The European Pulsar Timing Array and the Large European Array for Pulsars, Classical and Quantum Gravity 30 (Nov., 2013) 224009.

[25] H. J. Pletsch, L. Guillemot, H. Fehrmann, B. Allen, M. Kramer, C. Aulbert et al., Binary Millisecond Pulsar Discovery via Gamma-Ray Pulsations, Science 338 (Dec., 2012) 1314, [1211. 1385 ].

[26] T. J. Johnson, C. Venter, A. K. Harding, L. Guillemot, D. A. Smith, M. Kramer et al., Constraints on the Emission Geometries and Spin Evolution of Gamma-Ray Millisecond Pulsars, ApJ Suppl. 213 (July, 2014) 6, [1404.2264].

[27] P. A. Caraveo, Gamma-Ray Pulsar Revolution, Annual Review of Astronomy and Astrophysics 52 (Aug., 2014) 211-250, [1312.2913].

[28] I. A. Grenier and A. K. Harding, Gamma-ray pulsars: A gold mine, Comptes Rendus Physique 16 (Aug., 2015) 641-660, [1509.08823].

[29] A. A. Abdo, M. Ackermann, M. Ajello, W. B. Atwood, M. Axelsson, L. Baldini et al., The First Fermi Large Area Telescope Catalog of Gamma-ray Pulsars, ApJ Suppl. 187 (Apr., 2010) 460-494, [0910.1608]. 